\title{
Aurora-A as an independent molecular prognostic marker in gastric cancer
}

\author{
JING WANG ${ }^{1,5}$, SHENG YANG ${ }^{1}$, HAIZENG ZHANG $^{2}$, YAN SONG ${ }^{3}$, XUN ZHANG $^{3}$, \\ HAILI QIAN $^{4}$, XIAOHONG HAN $^{1}$ and YUANKAI SHI ${ }^{1}$ \\ Departments of ${ }^{1}$ Medical Oncology, ${ }^{2}$ Gastrointestinal Surgical Oncology and ${ }^{3}$ Pathology and \\ ${ }^{4}$ State Key Laboratory of Molecular Oncology, Cancer Institute and Hospital, Chinese Academy \\ of Medical Sciences and Peking Union Medical College, Beijing 100021, P.R. China
}

Received January 10, 2011; Accepted March 21, 2011

DOI: $10.3892 /$ or.2011.1250

\begin{abstract}
Aurora-A, encoding serine/threonine kinases with a key role in mitosis has been demonstrated to be involved in tumor progression. Hematogenous and lymphatic metastasis are also involved in cancer progression. Vascular endothelial growth factors (VEGFs) and their receptors (VEGFRs) have been implicated in tumor-related angiogenesis and lymphagenesis. The purpose of this study was to analyze the expression and prognostic significance of Aurora-A, VEGFs and VEGFRs in gastric cancer. The expression profiles of Aurora-A, VEGF-A and VEGF-D in gastric cancer cell lines were detected employing real-time reverse transcription polymerase chain reaction and Western blot analysis. The expression levels of Aurora-A, VEGF-A/VEGFR-2, VEGF-D/VEGFR-3 and clinicopathological characteristics were analyzed in 89 gastric cancer patients treated with curative surgery. Univariate analysis demonstrated that histological grade $(\mathrm{P}=0.052)$, TNM stage $(\mathrm{P}<0.001)$, lymphovascular involvement $(\mathrm{P}<0.001)$, Aurora- $\mathrm{A}(\mathrm{P}<0.001)$ and VEGF-D $(\mathrm{P}=0.048)$ were prognostic factors. The presence of Aurora-A was correlated with tumor progression $(\mathrm{P}=0.053)$ and shorter survival $(\mathrm{P}=0.001)$. Cox multivariate regression analysis demonstrated that Aurora-A positive expression, stage III-IV and lymphovascular involvement were independent unfavorable prognostic factors in gastric cancer. Aurora-A positive expression was predictive for worse outcome in patients without lymph node metastasis $(\mathrm{P}<0.05)$ and in patients with stage III-IV $(\mathrm{P}<0.001)$. Aurora-A could serve as an independent prognostic marker in gastric cancer and could identify patients with worse outcome even in a relatively early and local disease, thus offering valuable
\end{abstract}

Correspondence to: Dr Yuankai Shi or Dr Xiaohong Han, Department of Medical Oncology, Cancer Institute and Hospital, Chinese Academy of Medical Sciences and Peking Union Medical College, Beijing 100021, P.R. China

E-mail: syuankai@126.com; syuankai@yahoo.cn

E-mail: xiaohonghanmd@126.com

Present address: ${ }^{5}$ Department of Radiation Oncology, Air Force General Hospital, Beijing 100142, P.R. China

Key words: Aurora-A, prognostic marker, gastric cancer information for administering individualized treatment and/or surveillance for these patients.

\section{Introduction}

Gastric cancer is the fourth most common cancer (1) and the second leading cause of cancer death worldwide (2). According to the Ministry of Health in China, the mortality of gastric cancer was in the third place behind cancers of the lung and liver, with 24.71 per 100,000 in 2004-2005 (data not published). Surgery is the primary treatment for this disease, but the prognosis is still quite unsatisfactory with 5-year overall survival of less than $30 \%$ (3). Although recent studies have demonstrated that combined modality therapy based on surgery contributes to ameliorating survival (4-6), the optimal tailored treatment for high-risk patients remains unclear. Therefore, the identification of novel molecular prognostic markers is indispensable to implementing individualized treatment.

Aurora-A, a member of mitotic regulated serine/threonine kinases in process of chromosome segregation and cytokinesis, plays an important role in maintaining genome stability (7). The overexpression of Aurora-A has been observed in a variety of solid tumors (8) and most importantly, it has been demonstrated to be associated with tumor progression (9-12). An earlier study showed that overexpression of Aurora-A was strongly associated with aggressive behavior in bladder cancer (13). Further studies have demonstrated that Aurora-A status is associated not only with clinicopathological characteristics, but also with clinical outcomes in a series of cancers (14-17), indicating that Aurora-A has potential to be a molecular marker affecting cancer-specific survival. However, the correlation of Aurora-A with cancer progression and clinical outcome has not yet been reported in gastric cancer.

Because of important roles of angiogenesis and lymphangiogenesis in cancer progression $(18,19)$, the correlation of vascular endothelial growth factors (VEGFs) and their receptors (VEGFRs) with lymphangiogenic processes has been evaluated (20-23). The correlation of VEGFs and VEGFRs with lymphatic tumor spread, tumor progression as well as clinical outcome has been observed in gastric cancer $(24,25)$. Of notable interest, VEGF-D and VEGFR-3 have both been identified as independent prognostic markers in gastric cancer (26). 
In this study, to clarify the clinical significance of Aurora-A, VEGF-A/VEGFR-2 and VEGF-D/VEGFR-3 in gastric cancer, we determined transcription and translation levels of Aurora-A, VEGF-A and VEGF-D in gastric cancer cell lines, examined the protein and their receptors expression in gastric cancer tissues and adjacent normal tissues, analyzed the relationship between the status of Aurora-A, VEGF-A/VEGFR-2 as well as VEGF-D/VEGFR-3 and clinicopathological features and clinical outcomes in gastric cancer patients. We hypothesize that Aurora-A could be an important prognostic factor and a preferable molecular predictive marker, reflecting cancer progression and clinical outcomes in gastric cancer.

\section{Materials and methods}

Patient selection and tissue specimens. Between March 1999 and December 2000, the data of 335 gastric patients, who were treated with curative surgery, was accrued in Cancer Hospital, Chinese Academy of Medical Sciences and Peking Union Medical College, Beijing, China. Among them, clinical data and tissues of 89 consecutive patients were involved in our study. These patients were not treated with additional anticancer therapy except curative surgery. Patients with remnant gastric cancer and the secondary tumor were not enrolled in this study. R0 resection is described as a complete resection with negative margins, including proximal or distal margins of $\geq 4 \mathrm{~cm}$ from the gross tumor, omental bursa along with the front leaf of transverse mesocolon. The extent of lymph node dissection described as D2 dissection involves perigastric lymph nodes, greater and lesser omental lymph nodes (station 1-6), the nodes along the corresponding arteries (station 7-11) except splenectomy. R0 resection and D2 dissection are described as the curative surgery. All cases were diagnosed as gastric adenocarcinoma. Aurora-A was analyzed in 89 cases. For the limitation of tissue specimens, VEGF-A was analyzed in 88 cases, VEGF-D in 85 cases, VEGFR-2 in 86 cases and VEGFR-3 in 88 cases. The median age for all patients was 66 years, ranging from 31 to 85 years. The median follow-up time was 93 months. Up until the last follow-up, 61 patients died, 27 patients survived free of disease, and 1 patient survived with lymph node metastasis. Staging and diagnosis of gastric carcinoma was assessed according to the AJCC Cancer Staging Manual (27). Written informed consent was obtained from all patients before any study-related procedure was performed.

Cell culture. Gastric cancer (GC) cell lines from both primary tumor and metastatic foci with different grades of differentiation such as AGS, MGC803, MKN45, BGC823, SUN-5, N87, SNU-16 and SGC7901 were cultured in RPMI-1640 medium supplemented with $10 \%$ fetal bovine serum (FBS), penicillin 100 units $/ \mathrm{ml}$, streptomycin $100 \mu \mathrm{g} / \mathrm{ml}$ and L-glutamine $2 \mathrm{mM} / 1$. The cell lines were maintained at $37^{\circ} \mathrm{C}$ under $5 \%$ $\mathrm{CO}_{2}$ and saturated moisture.

Western blot assay. Western blot analysis was performed as described previously (28). The primary antibodies used are shown as follows: Aurora-A (dilution, 1:250), polyclonal rabbit (Abcam, Ab1287); VEGF-A (dilution, 1:100), polyclonal rabbit (Santa Cruz Biotechnology Inc., CA, sc-152); VEGF-D (dilution, 1:100), polyclonal goat (Santa Cruz Biotechnology
Inc., sc-7602). Anti- $\beta$-actin mouse monoclonal antibody (dilution, 1:1000; Santa Cruz Biotechnology Inc.) was used as an internal loading control. The secondary antibodies of anti-rabbit, anti-goat and anti-mouse immunoglobulin G conjugated to horseradish peroxidase were obtained from Santa Cruz Biotechnology Inc. All proteins were visualized using an enhanced chemiluminescence detection (Millipore, MA, USA).

Quantitative mRNA determinations. Total RNA was extracted from GC cell lines with TRIzol reagent (Invitrogen, CA, USA) and reversely transcribed with Vigoscript First Strand cDNA Synthesis Kit (Vigorous, Beijing, China) according to the manufacturer's protocol. The housekeeping gene $\beta$-actin was used as an internal quantitative control. The gene-specific primers for real-time quantitative reverse transcription polymerase chain reaction (RT-PCR) are as follows: Aurora-A forward (5'-3') ACTCAGCAATTTCCTTGTCAGA, reverse (5'-3') GATTATTTTCAGGTGCCGATG; VEGF-A forward (5'-3') CTCACCAAGGCCAGCACATAGG, reverse (5'-3') ATCTGGTTCCGAAAACCCTGAG; VEGF-D forward (5'-3') CTGGAACAGAAGACCACTCTCATC, reverse (5'-3') CTCGCAACGATCTTCGTCAAA; $\beta$-actin forward (5'-3') TGCATTGTTACAGGAAGTCCCTT, reverse (5'-3') GGGAGAGGACTGGGCCAT. cDNAs generated from $30 \mathrm{ng}$ of total RNA were used for real-time quantification. The SYBR Premix Ex Taq assay (Takara, Dalian, China) and the ABI PRISM 7300 sequence detection system (Applied Biosystems) were used for detecting real-time quantitative PCR products. PCRs for each sample were done in triplicate for both target gene and $\beta$-actin control. The cycling conditions were as follows: stage $1,95^{\circ} \mathrm{C}$ for $10 \mathrm{sec}$; stage 2 , 40 cycles of amplification at $95^{\circ} \mathrm{C}$ for $5 \mathrm{sec}$ and annealing temperature for $40 \mathrm{sec}$; stage $3,95^{\circ} \mathrm{C}$ for $15 \mathrm{sec}, 60^{\circ} \mathrm{C}$ for $20 \mathrm{sec}, 95^{\circ} \mathrm{C}$ for $15 \mathrm{sec}$. The annealing temperature for both Aurora-A and VEGF-D was $57^{\circ} \mathrm{C}$ and $59^{\circ} \mathrm{C}$ for VEGF-A. The expression levels of target genes and the housekeeping gene were analyzed using the $2^{-\Delta \Delta \mathrm{CT}}$ method as described previously (29).

Immunohistochemistry analysis. Immunohistochemical staining was performed as described previously (9). The staining results obtained from the given primary antibodies as described above were confirmed by three separate determinations. VEGFR-2 (sc-6251) and VEGFR-3 (sc-321) antibodies from Santa Cruz Biotechnology Inc. were also assessed (VEGFR-2, 1:100; VEGFR-3, 1:50). The semi-quantitative evaluation of immunohistochemical staining was employed according to staining intensity and staining extent. The staining intensity was rated as negative, weak, moderate and strong respectively. The staining extent was classified according to percentage of epithelial cells showing specific immunoreactivity as follows: negative (no immunoreactivity), weak ( $<10 \%$ positive cells), moderate (10-50\% positive cells), strong ( $>50 \%$ positive cells). The corresponding score was recorded as $0,1,2$ and 3 respectively. The comprehensive assessment obtained from staining intensity and extent included negative (score 0 ), weak (score 1-2), moderate (score 3-4) and strong (score 5-6). Only samples showing moderate or strong immunoreactivity were considered to be positive expression (score 3-6). For VEGF-A, overexpres- 
A

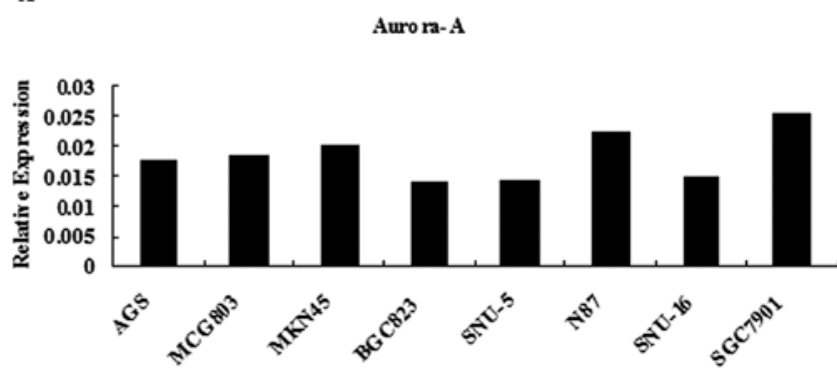

B

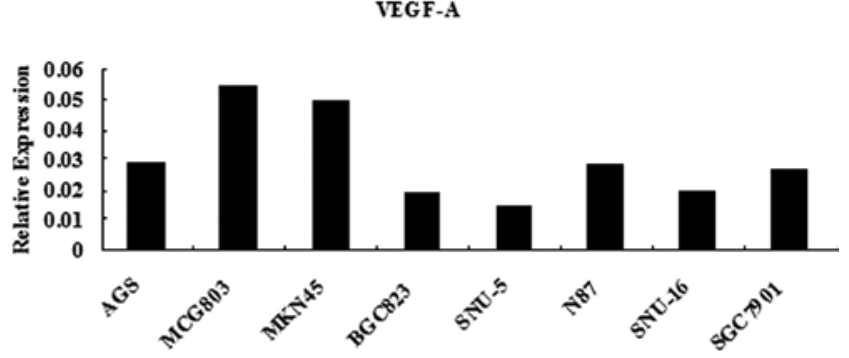

C

VEGF-D

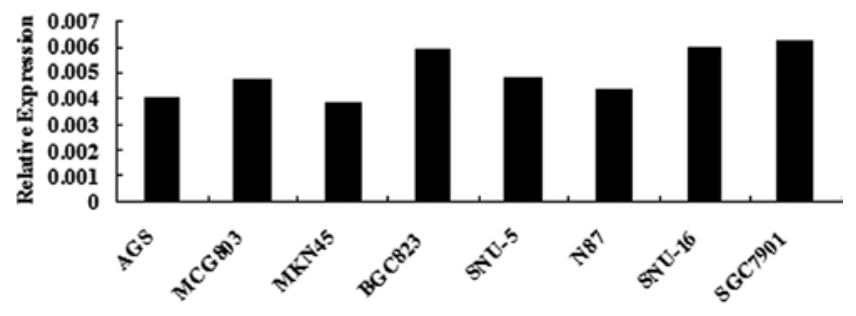

Figure 1. Real-time reverse transcription polymerase chain reaction determination of Aurora-A (A), VEGF-A (B) and VEGF-D (C) mRNA levels in diverse gastric cancer cell lines. The data shown represent typical results obtained in a series of three independent experiments.

sion was described as strong immunoreactivity (score 5-6), and low/negative expression included negative, weak and moderate immunoreactivity (score 0-4). The positive control staining according to the instructions of products was as follows: Aurora-A in testis tumor tissues, VEGF-A and VEGFR-3 in breast cancer tissues, VEGFR-2 in colon cancer tissues. The strongly positive expression of VEGF-D in the tumor tissues was used as its own positive control staining. The phosphatebuffered saline (PBS) substitution for primary antibodies was used as a negative control staining. The results of immunohistochemistry staining were scored independently by two expert pathologists. Consistent assessments were regarded as results of immunohistochemistry. When the discrepancy occurred in assessments, consensuses achieved in discussion were adopted as results of immunohistochemistry.

Statistical analysis. Comparison of the qualitative data was done by $\chi^{2}$ test. Relationships between positivity for Aurora-A, VEGF-A/VEGFR-2, VEGF-D/VEGFR-3 and clinicopathological features were evaluated using Spearman's rank correlation coefficient. Overall survival (OS) and disease-free survival (DFS) were estimated by the product-limit KaplanMeier method. OS was measured from the date of curative
AGS MGCS03 MKN45 BGCS23 SNU-5NS7 SNU-16 SGC7901
A Aurora-A

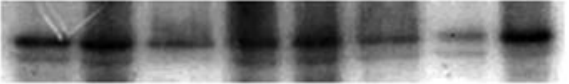

B

VEGF-A

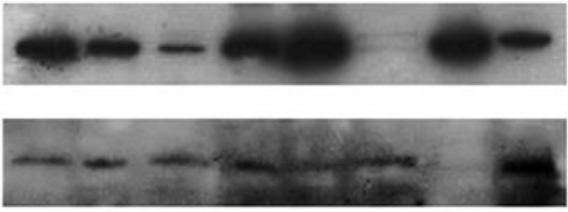

$42 \mathrm{kDa}$

C VEGF-D

D $\beta$-Actin

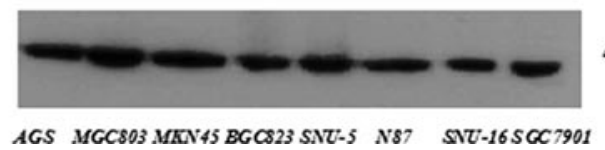

$21 \mathrm{kDa}$
Figure 2. Results of Western blot analysis of Aurora-A (A), VEGF-A (B) and VEGF-D (C) protein expression in gastric cancer cell lines.

surgery until time of death of any causes, or until last followup. DFS was measured from the date of curative surgery until time of local relapse or distant metastasis, or until last follow-up, or death. The differences between survival curves were calculated by the log-rank test. Multivariate analysis was performed using the Cox model.

\section{Results}

Aurora-A, VEGF-A and VEGF-D $m R N A$ and protein levels in $G C$ cell lines. The real-time RT-PCR detection demonstrated that the mRNA levels of Aurora-A, VEGF-A and VEGF-D varied with the cell lines (Fig. 1). Aurora-A mRNA had relatively high expression in well and moderately differentiated type such as N87 and SGC803 cell lines (Fig. 1A). High VEGF-A gene transcription was observed in poorly differentiated type such as MGC803 and MKN45 cell lines (Fig. 1B). Higher VEGF-D mRNA was more commonly seen in poorly and moderately differentiated types such as in BGC823, SNU-16 and SGC7901 cell lines (Fig. 1C). Western blot analysis revealed that the protein levels of Aurora-A, VEGF-A and VEGF-D were expressed in GC cell lines (Fig. 2). Aurora-A protein was expressed in all GC cell lines with different levels, basically consistent with the counterpart mRNA levels (Fig. 2A). VEGF-A protein expression was relatively high in a majority of GC cell lines, whereas it was lowly expressed in N87 cell line (Fig. 2B). VEGF-D protein expression was low in the SNU-16 cell line (Fig. 2C).

Aurora-A, VEGF-A and VEGF-D in GC tissues. Immunohistochemistry analysis of Aurora-A, VEGF-A/VEGFR-2 and VEGF-D/VEGFR-3 protein were performed in GC tissues and adjacent normal tissues $(n=89)$ (Fig. 3). In contrast to the normal tissues, Aurora-A, VEGF-A and VEGF-D were significantly highly expressed in cancer tissues $(\mathrm{P}<0.001)$ (Table I), indicating an active role in carcinogenesis and as a potential marker differentiating normal and cancer tissues.

Correlation analysis and clinicopathological characteristics. The median DFS and OS were 16.7 months (1.0-54.9 months) and 28.3 months (1.0-104.1 months), respectively. Table II shows the correlation between the positive expression of Aurora-A, VEGF-D, VEGF-A and clinicopathological char- 
A
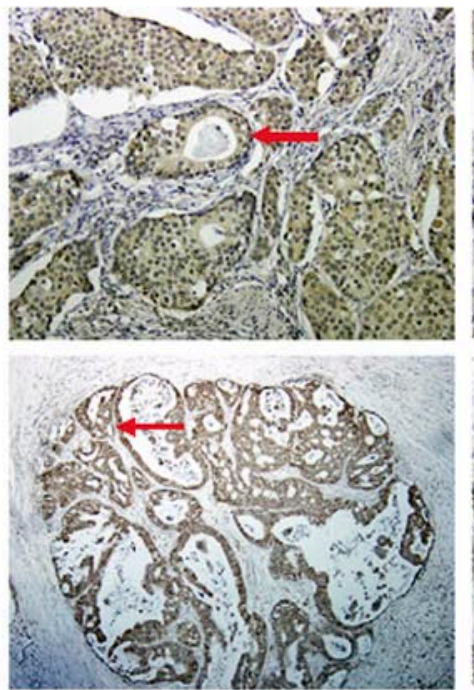

C

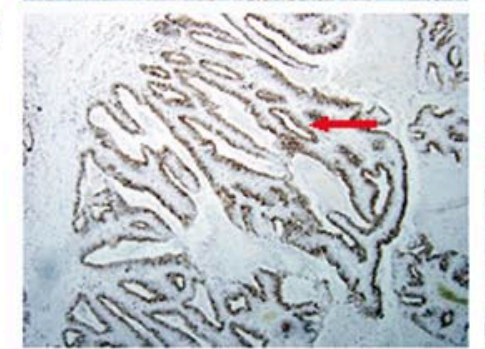

D

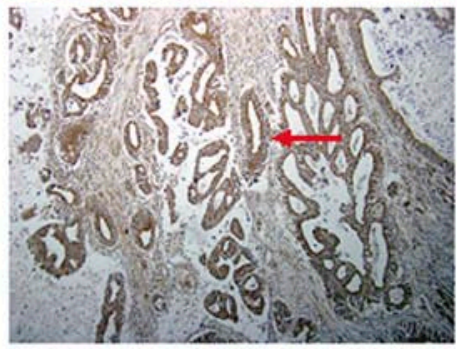

$\mathbf{E}$

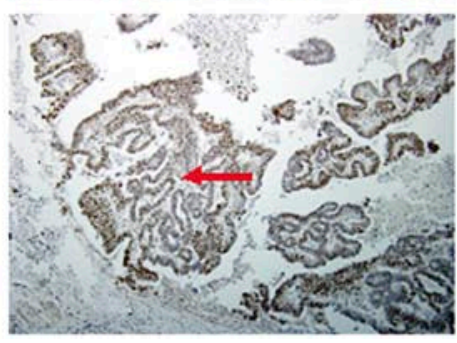

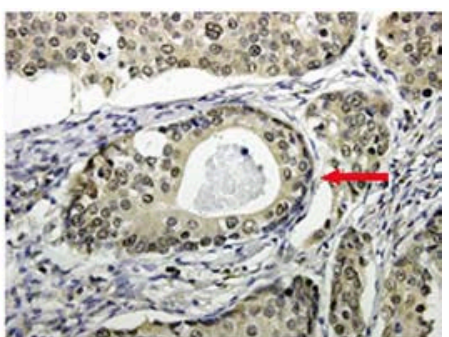
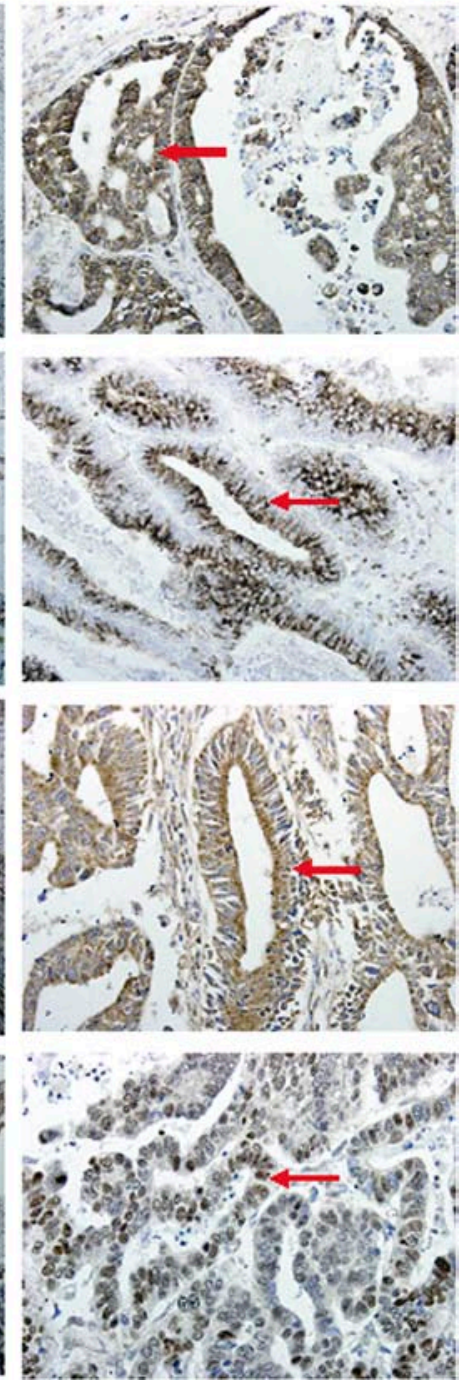

Figure 3. Representative results of immunohistochemistry analysis for Aurora-A (A), VEGF-A (B), VEGF-D (C) and VEGF-R2 (D) and VEGF-R3 (E) proteins in gastric cancer tissues. The arrows shown gastric cancer tissues in the left (x100) were magnified in the right (x400).

Table I. Positive expression levels of the biomarkers in gastric cancer.

\begin{tabular}{lccccccr}
\hline & & \multicolumn{2}{c}{ Tumor } & & \multicolumn{2}{c}{ Normal } & \\
Biomarker & No. & $\mathrm{N}$ & $(\%)$ & & $\mathrm{N}$ & $(\%)$ & P-value \\
\hline Aurora-A & 89 & 51 & 57.3 & & 9 & 10.1 & $<0.001$ \\
VEGF-A & 88 & 54 & 61.4 & & 17 & 19.3 & $<0.001$ \\
VEGF-D & 85 & 65 & 76.5 & & 10 & 11.8 & $<0.001$ \\
VEGFR-2 & 86 & 68 & 79.1 & & 31 & 45.6 & $<0.001$ \\
VEGFR-3 & 88 & 30 & 34.1 & & 20 & 22.7 & 0.095 \\
\hline
\end{tabular}

VEGF, vascular endothelial growth factor; VEGFR, VEGF receptor. acteristics in 89 gastric cancer patients treated with curative surgery. The positive expression of Aurora-A protein showed a close association with pathological TNM stage $(\mathrm{P}=0.053)$. There was no significant correlation between the positive expression of VEGF-A and clinicopathological characteristics. There was a significant correlation between the positive expression of VEGF-D and age $(\mathrm{P}=0.021)$, $\mathrm{T}$ stage $(\mathrm{P}<0.001), \mathrm{N}$ stage $(\mathrm{P}=0.013)$ and pathological TNM stage $(\mathrm{P}=0.004)$.

Prognostic significance of Aurora-A, VEGF-A and VEGF-D. To elucidate the prognostic significance of Aurora-A, VEGF-A and VEGF-D in gastric cancer, clinicopathological characteristics and the molecular markers were analyzed in 89 gastric 
Table II. Correlation of Aurora-A, VEGF-A and VEGF-D immunochemistry with clinicopathological characteristics in patients with curative surgery in gastric cancer.

\begin{tabular}{|c|c|c|c|c|c|c|c|c|c|c|c|c|}
\hline \multirow[b]{2}{*}{ Characteristics } & \multicolumn{4}{|c|}{ Aurora-A positive } & \multicolumn{4}{|c|}{ VEGF-A positive } & \multicolumn{4}{|c|}{ VEGF-D positive } \\
\hline & No. & $\mathrm{N}$ & $(\%)$ & P-value & No. & $\mathrm{N}$ & $(\%)$ & P-value & No. & $\mathrm{N}$ & $(\%)$ & P-value \\
\hline Gender & & & & 0.846 & & & & & & & & 0.288 \\
\hline Male & 78 & 45 & 57.7 & & 77 & 46 & 59.7 & 0.414 & 75 & 56 & 74.7 & \\
\hline Female & 11 & 6 & 54.5 & & 11 & 8 & 72.7 & & 10 & 9 & 90.0 & \\
\hline Age (years) & & & & 0.676 & & & & & & & & 0.021 \\
\hline$\leq 60$ & 26 & 14 & 53.8 & & 26 & 13 & 50.0 & 0.160 & 25 & 15 & 60.0 & \\
\hline$>60$ & 63 & 37 & 58.7 & & 62 & 41 & 66.1 & & 60 & 50 & 83.3 & \\
\hline Tumor site & & & & 0.376 & & & & & & & & 0.118 \\
\hline Proximal & 49 & 26 & 53.1 & & 49 & 34 & 69.4 & 0.085 & 47 & 39 & 83.0 & \\
\hline Non-proximal & 40 & 25 & 62.5 & & 39 & 20 & 51.3 & & 38 & 26 & 68.4 & \\
\hline Histological grade & & & & 0.834 & & & & & & & & 0.807 \\
\hline Well-moderately & 34 & 19 & 55.9 & & 34 & 24 & 70.6 & 0.162 & 32 & 24 & 75.0 & \\
\hline Poorly-undifferentiated & 55 & 32 & 58.2 & & 54 & 30 & 55.6 & & 53 & 41 & 77.4 & \\
\hline Tumor infiltration & & & & 0.110 & & & & & & & & $<0.001$ \\
\hline $\mathrm{T} 1$ & 18 & 7 & 38.9 & & 18 & 8 & 44.4 & 0.125 & 18 & 8 & 44.4 & \\
\hline $\mathrm{T} 2$ & 3 & 2 & 66.7 & & 3 & 2 & 66.7 & & 2 & 1 & 50.0 & \\
\hline $\mathrm{T} 3$ & 68 & 42 & 61.8 & & 67 & 44 & 65.7 & & 65 & 56 & 86.2 & \\
\hline Lymph node metastasis & & & & 0.083 & & & & & & & & 0.013 \\
\hline No & 25 & 12 & 48.0 & & 25 & 12 & 48.0 & 0.313 & 24 & 14 & 58.3 & \\
\hline N1 & 37 & 20 & 54.1 & & 37 & 25 & 67.6 & & 35 & 28 & 80.0 & \\
\hline $\mathrm{N} 2$ & 17 & 11 & 64.7 & & 17 & 13 & 76.5 & & 16 & 14 & 87.5 & \\
\hline N3 & 10 & 8 & 80.0 & & 9 & 4 & 44.4 & & 10 & 9 & 90.0 & \\
\hline TNM stage & & & & 0.053 & & & & & & & & 0.004 \\
\hline IA & 16 & 6 & 37.5 & & 16 & 7 & 43.8 & 0.277 & 16 & 7 & 43.8 & \\
\hline IB & 2 & 1 & 50.0 & & 2 & 2 & 100.0 & & 1 & 1 & 100.0 & \\
\hline II & 9 & 6 & 66.7 & & 9 & 4 & 44.4 & & 9 & 7 & 77.8 & \\
\hline IIIA & 35 & 19 & 54.3 & & 35 & 24 & 68.6 & & 33 & 27 & 81.8 & \\
\hline IIIB & 17 & 11 & 64.7 & & 17 & 13 & 76.5 & & 16 & 14 & 87.5 & \\
\hline IV & 10 & 8 & 80.0 & & 9 & 4 & 44.4 & & 10 & 9 & 90.0 & \\
\hline & & & & 0.254 & & & & & & & & 0.006 \\
\hline I-II & 27 & 13 & 48.1 & & 27 & 13 & 48.1 & 0.092 & 26 & 15 & 57.7 & \\
\hline III-IV & 62 & 38 & 61.3 & & 61 & 41 & 67.2 & & 59 & 50 & 84.7 & \\
\hline $\begin{array}{l}\text { Lymphovascular } \\
\text { involvement }\end{array}$ & & & & 0.187 & & & & & & & & 0.898 \\
\hline Present & 12 & 9 & 75.0 & & 11 & 9 & 81.8 & 0.140 & 12 & 9 & 75.0 & \\
\hline Absent & 77 & 42 & 28.6 & & 77 & 45 & 58.4 & & 73 & 56 & 76.7 & \\
\hline
\end{tabular}

The bold indicates statistically significant correlation. Results were calculated by Spearman's rank correlation coefficient. VEGF, vascular endothelial growth factor.

cancer patients with curative surgery. Univariate analysis demonstrated that histological grade $(\mathrm{P}=0.052)$ (Fig. 4A), TNM stage $(\mathrm{P}<0.001)$ (Fig. 4B) and lymphovascular involvement $(\mathrm{P}<0.001)$ (Fig. 4C) were clinicopathological factors affecting survival; Aurora-A and VEGF-D were molecular prognostic factors $(\mathrm{P}<0.001$ and 0.048 , respectively) (Fig. 4D and $F$ ). There was no significant correlation between the positive expression of VEGF-A, VEGFR-2, VEGFR-3 and survival. In contrast to patients with low and negative expression of VEGF-A, the patients with overexpression of VEGF-A had a worse survival $(\mathrm{P}=0.029)$ (Fig. 4E). Cox regression analysis demonstrated that Aurora-A positive expression, stage III-IV and lymphovascular involvement were independent unfavorable prognostic factors (Table III). 
A

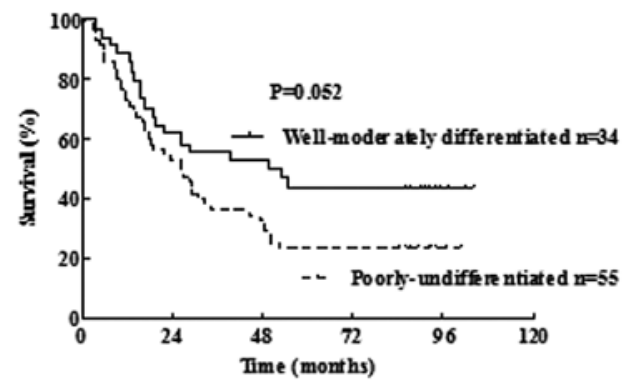

C

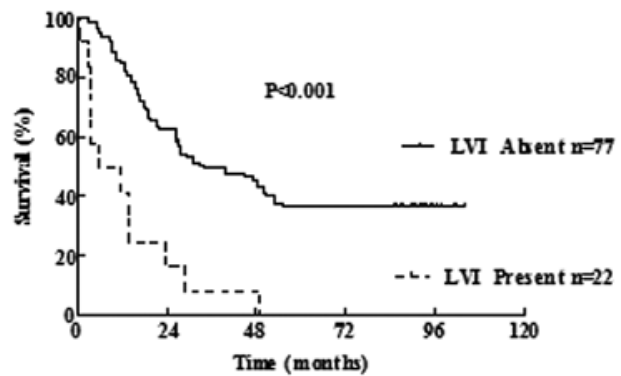

$\mathbf{E}$

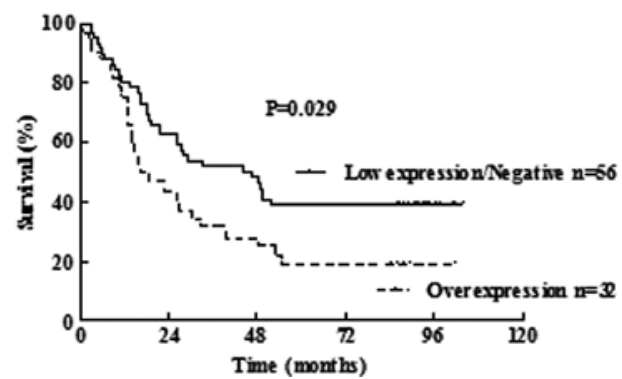

B

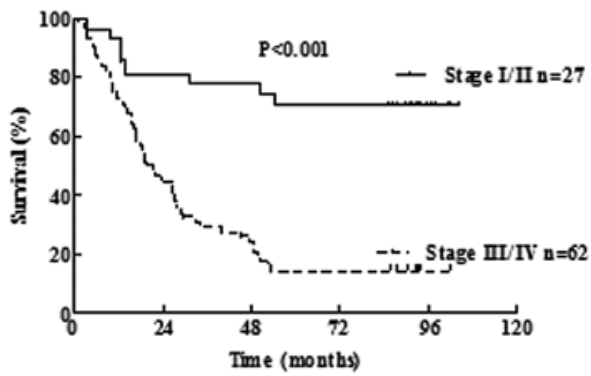

D

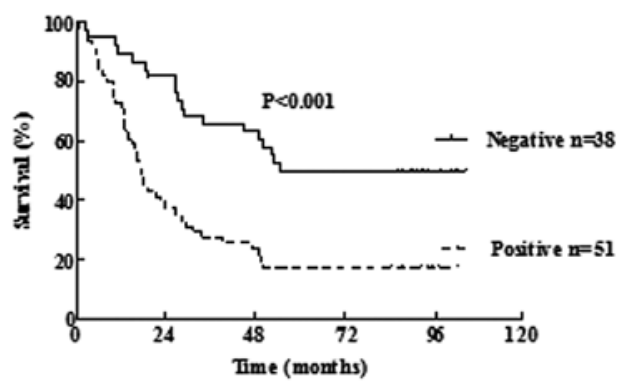

$\mathbf{F}$

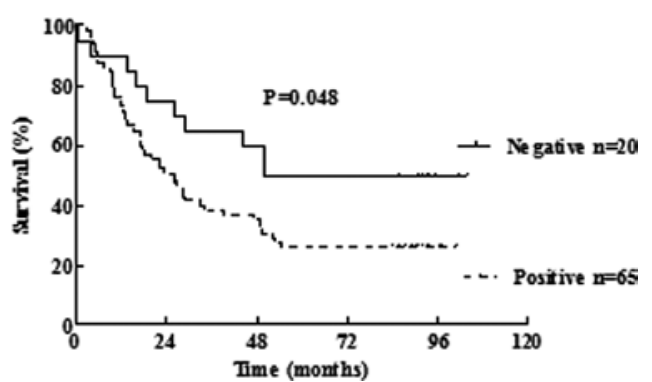

Figure 4. Relationships between histological grade (A), TNM stage (B), lymphovascular involvement (LVI) (C), immunohistochemistery expression for Aurora-A (D), VEGF-A (E), VEGF-D (F) and carcinoma-specific survival were evaluated using Kaplan-meier method. Comparison of survival curves were performed using log-rank test.

Predictive value of Aurora-A in gastric cancer. To clarify the predictive value of Aurora-A as an independent molecular prognostic factor in gastric cancer with curative surgery, the intensive analysis was performed to discriminate high-risk patients over strata of clinicopathological characteristics. In the subset analysis of lymph node metastases $\left(\mathrm{pN}^{+}\right)$, Aurora-A could show a significant survival difference in patients with $\mathrm{pN} 0$ and $\mathrm{pN} 1(\mathrm{P}<0.05)$ (Fig. 5A and $\mathrm{B})$. Owing to lymphovascular involvement and stage III/IV as an independent unfavorable prognostic factor, the significance of Aurora-A was also analyzed. Compared with patients with Aurora-A negative expression, Aurora-A positive expression indicated a worse outcome in patients without lymphovascular involvement and in patients with stage III/ IV ( $\mathrm{P}<0.001)$ (Fig. 5C and D).

\section{Discussion}

As far as we know, the present study is the first report on Aurora-A as an independent molecular prognostic factor in gastric cancer patients treated with radical surgery. Firstly, expression of Aurora-A in transcriptional and protein level varied with gastric cancer cell lines, to some extent, reflecting the innate characteristics in cell phenotypes. Secondly, there was significant expression difference of Aurora-A between cancer tissues and the adjacent normal tissues $(\mathrm{P}<0.001)$. Thirdly, Aurora-A protein was significantly associated with TNM stage (Table II). More importantly, Aurora-A as an independent molecular prognostic factor that identified a worse outcome in patients with pNO (Fig. 5A) and pN1 (Fig. 5B). Also, Aurora-A identified unfavorable outcome in patients without lymphovascular involvement (Fig. 5C) and in patients with stage III/IV (Fig. 5D). These results further demonstrated that Aurora-A could offer valuable prognostic information in relative early and local advanced gastric cancer.

The correlation of Aurora-A status with malignant phenotype and tumor progression has been evaluated in a series of studies. The earlier studies revealed that Aurora-A expression was involved with aneuploid formation, differentiated type and poor prognosis in gastric cancer $(30,31)$. Similarly, 
Table III. Determination of independent factors affecting carcinoma-specific and disease-free survival in patients treated with curative surgery in gastric cancer.

\begin{tabular}{|c|c|c|c|c|c|c|}
\hline \multirow[b]{2}{*}{ Prognostic factor } & \multicolumn{3}{|c|}{ Carcinoma-specific survival } & \multicolumn{3}{|c|}{ Disease-free survival } \\
\hline & $\mathrm{RR}$ & $95 \% \mathrm{CI}$ & $\mathrm{P}$-value & $\mathrm{RR}$ & $95 \% \mathrm{CI}$ & P-value \\
\hline Aurora-A & & & 0.001 & & & 0.001 \\
\hline Negative & 1 & & & 1 & & \\
\hline Positive & 2.923 & $1.577-5.416$ & & 2.941 & $1.585-5.457$ & \\
\hline VEGF-A & & & 0.231 & & & 0.322 \\
\hline Low expression/negative & 1 & & & 1 & & \\
\hline Overexpression & 1.417 & $0.801-2.507$ & & 1.338 & $0.752-2.378$ & \\
\hline VEGF-D & & & 0.675 & & & 0.764 \\
\hline Negative & 1 & & & 1 & & \\
\hline Positive & 1.170 & $0.561-2.441$ & & 1.119 & $0.536-2.338$ & \\
\hline Histological grade & & & 0.158 & & & 0.255 \\
\hline Well / moderately & 1 & & & 1 & & \\
\hline Poorly-undifferentiated & 1.532 & $0.848-2.767$ & & 1.419 & $0.777-2.591$ & \\
\hline Stage & & & $<0.001$ & & & $<0.001$ \\
\hline I-II & 1 & & & 1 & & \\
\hline III-IV & 5.298 & $2.256-12.441$ & & 5.234 & $2.216-12.359$ & \\
\hline Lymphovascular involvement & & & 0.007 & & & 0.013 \\
\hline Present & 1 & & & 1 & & \\
\hline Absent & 2.665 & $1.300-5.461$ & & 2.495 & $1.216-5.119$ & \\
\hline
\end{tabular}

The bold indicates statistically significant results. RR, ratio of relative risk of death; CI, confidence interval; VEGF, vascular endothelial growth factor.

A

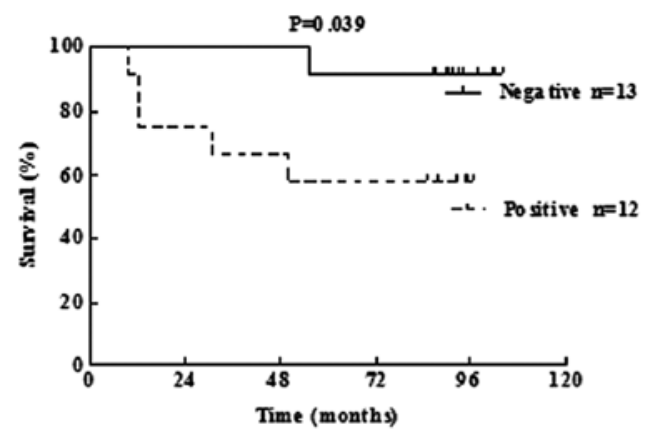

C

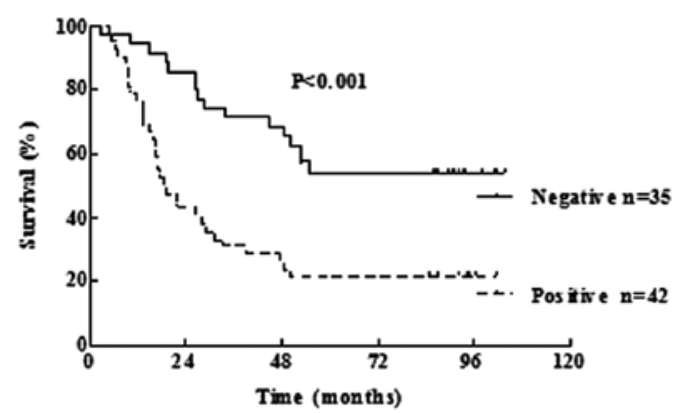

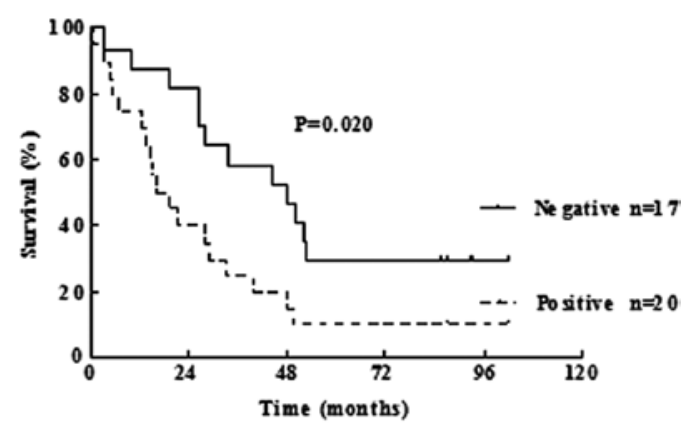

D

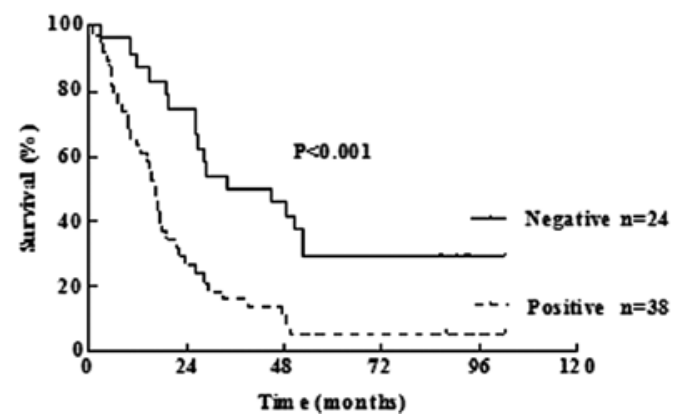

Figure 5. Comparisons of survival curves between Aurora-A negative and positive expression in pN0 (A) and pN1, in absence of lymphovascular involvement (C) and in stage III/IV (D). 
Table IV. Correlation between Aurora-A and clinicopathological characteristics, prognosis in tumors.

\begin{tabular}{lcrllll}
\hline Author & Time & \multicolumn{1}{c}{ Tumor } & No. & $\begin{array}{c}\text { Clinicopathologic } \\
\text { characteristics }\end{array}$ & \multicolumn{1}{c}{ Treatment } & Prognosis \\
\hline Sen et al $(13)$ & 2002 & Bladder cancer & 205 & T category, grade, stage & No adjuvant therapy & DFS, OS \\
Tanaka et al $(14)$ & 2005 & ESCC & 142 & T category, LNM & Preoperative chemotherapy & DFS, OS \\
Reiter et al $(15)$ & 2006 & HNSCC & 66 & LNM, DM, stage & Unknown & DFS, OS \\
Ogawa et al $(17)$ & 2008 & NSCLC & 189 & Stage & Postoperative chemotherapy & OS \\
Landen et al $(38)$ & 2007 & Ovarian cancer & 70 & & Adjuvant therapy & OS \\
Mendiola et al $(39)$ & 2009 & Ovarian cancer & 68 & Unknown & Postoperative chemotherapy & PFS, OS \\
Zhang et al $(40)$ & 2009 & Cervical cancer & 74 & Stage, grade, LNM, HM, PM & Unknown & DFS, OS \\
Nalder et al $(41)$ & 2008 & Breast cancer & 533 & Grade, PR, LNM & No adjuvant therapy & OS \\
Shang et al $(42)$ & 2009 & Neuroblastoma & 67 & Stage, histology & Unknown & PFS \\
Scarpini et al $(43)$ & 2010 & Urothelial cancer & 42 & Vascular invasion & Unknown & RFS \\
Wang et al & Present & Gastric cancer & 89 & Stage & No adjuvant therapy & DFS, OS \\
\hline
\end{tabular}

HNSCC, head and neck squamous cell cancer; ESCC, esophageal squamous cell cancer; NSCLC, non-small cell lung cancer. DFS, disease-free survival; OS, overall survival; LNM, lymph node metastasis; DM, distant metastasis; PFS, progression-free survival; HM, hematogenous metastasis; PM, parametrial invasion; PR, progesterone receptor. ${ }^{a}$ Except for Tamoxifen; RFS, relapse-free survival.

Aurora-A was also found to be differentially expressed and regulated in colorectal cancers (32). Moreover, several studies reported that there was a close association between Aurora-A overexpression and malignant biological behavior of cancer progression (9,16,33-35). Additionally, Aurora-A overexpression was observed to be associated with EGFR activation and overexpression $(36,37)$. These studies support our results that Aurora-A as a molecular marker representing invasive differences in malignant phenotypes and could serve as a prognostic factor reflecting the extent of cancer progression in gastric cancer.

Similar to previous reports in bladder cancer (13), esophageal squamous cell carcinoma (14), head and neck squamous cell carcinoma (15), non-small cell lung cancer (17), ovarian cancer $(38,39)$, cervical cancer $(40)$, breast cancer (41), neuroblastoma (42) and urothelial carcinoma (43) (Table IV), our results demonstrate that Aurora-A is an independent molecular prognostic marker in gastric cancer. The presence of Aurora-A identified poor clinical outcome in patients with pN0 and pN1 $(\mathrm{P}<0.05)$ (Fig. 5A and B), in patients without lymphovascular involvement $(\mathrm{P}<0.001)$ (Fig. $5 \mathrm{C})$, even in patients with stage III/IV $(\mathrm{P}<0.001)$ (Fig. 5D). These results offer important and helpful information to indicate subsequent individualized treatment in patients receiving curative surgery gastric cancer, even in patients with relatively early and local advanced disease.

In our series, VEGF-D significantly correlates with $\mathrm{T}$ category, N category and TNM stage (Table II), and is demonstrated to be an unfavorable prognostic factor in univariate analysis $(\mathrm{P}=0.048)$. Our results are in agreement with previous clinical studies that VEGF-D is associated with lymphatic tumor spread, tumor progression and clinical outcome in gastric cancer $(24,26,44)$. However, VEGF-D is not an independent prognostic factor when Aurora-A and VEGF-A are incorporated into multivariate analysis (Table III). Juttner et al (26) reported that VEGF-D, VEGFR-3 and venous invasion were independent unfavorable prognostic factors in gastric cancer. Unfortunately, pathological stage regarded as a 'gold criteria' in evaluating outcome was not analyzed in the Cox hazard model. Compared with VEGFs/VEGFRs, Aurora-A seems to be a more informative prognostic marker in our study.

In addition to as a prognostic marker, Aurora-A has potential to be as a therapeutic target in individualized treatment. A previous study revealed that the treatment targeting Aurora-A kinase could suppress the tumor growth and enhance the taxane chemosensitivity (45). A further study demonstrated that Aurora-A served as a potential therapeutic target predicating outcomes in the patients receiving taxanebased and non-taxane-based chemotherapy (46). Recent studies suggested that Aurora-A kinase inhibitors showed antitumor activity alone and in combination with docetaxel $(47,48)$.

Given the evidence of the tumor progression and lymphatic tumor spread affecting survival, the independent molecular prognostic marker reflecting more invasive malignant phenotype, the improved survival available after targeting treatment against the molecular, Aurora-A is shown as a molecular prognostic marker and as a potential predictive marker in gastric cancer. For those patients with high risk of tumor progression and lymphatic tumor spread, combined modality should be considered for patients receiving curative-intent surgery. Taxane-based chemotherapy and Aurora-A kinase inhibitors should be considered to be as a therapeutic option for patients with Aurora-A positive expression. Observation only would be advisable for low risk of death and Aurora-A negative expression in the patients receiving radical surgery. In summary, Aurora-A is demonstrated to be a molecular prognostic marker for gastric cancer patients with curative surgery, which offers valuable prognostic information to administer individualized treatment and/or surveillance in the future. 


\section{Acknowledgements}

We thank Dr Jian Pan, Dr Lichao Sun and Dr Lizhao Chen, from Department of State Key Laboratory of Molecular Oncology, Cancer Institute and Hospital, Chinese Academy of Medical Sciences and Peking Union Medical College, for culturing and providing the gastric cancer cell lines and their technical assistance. This study was supported by Key Project of Ministry of Human Resources and Social Security of The People's Republic of China.

\section{References}

1. Kamangar F, Dores GM and Anderson WF: Patterns of cancer incidence, mortality, and prevalence across five continents: defining priorities to reduce cancer disparities in different geographic regions of the world. J Clin Oncol 24: 2137-2150, 2006.

2. Parkin DM, Bray F, Ferlay J and Pisani P: Global cancer statistics, 2002. CA Cancer J Clin 55: 74-108, 2005.

3. Hohenberger P and Gretschel S: Gastric cancer. Lancet 362: 305-315, 2003

4. Cunningham D, Allum WH, Stenning SP, et al: Perioperative chemotherapy versus surgery alone for resectable gastroesophageal cancer. N Engl J Med 355: 11-20, 2006.

5. Nakajima T, Kinoshita T, Nashimoto A, et al: Randomized controlled trial of adjuvant uracil-tegafur versus surgery alone for serosa-negative, locally advanced gastric cancer. Br J Surg 94: 1468-1476, 2007.

6. Sun P, Xiang JB and Chen ZY: Meta-analysis of adjuvant chemotherapy after radical surgery for advanced gastric cancer. Br J Surg 96: 26-33, 2009.

7. Marumoto T, Zhang D and Saya H: Aurora-A - a guardian of poles. Nat Rev Cancer 5: 42-50, 2005.

8. Fu J, Bian M, Jiang Q and Zhang C: Roles of Aurora kinases in mitosis and tumorigenesis. Mol Cancer Res 5: 1-10, 2007.

9. Tong T, Zhong Y, Kong J, et al: Overexpression of Aurora-A contributes to malignant development of human esophageal squamous cell carcinoma. Clin Cancer Res 10: 7304-7310, 2004.

10. Kulkarni AA, Loddo M, Leo E, et al: DNA replication licensing factors and aurora kinases are linked to aneuploidy and clinica outcome in epithelial ovarian carcinoma. Clin Cancer Res 13: 6153-6161, 2007

11. Torchia EC, Chen Y, Sheng H, et al: A genetic variant of Aurora kinase A promotes genomic instability leading to highly malignant skin tumors. Cancer Res 69: 7207-7215, 2009.

12. Loddo M, Kingsbury SR, Rashid M, et al: Cell-cycle-phase progression analysis identifies unique phenotypes of major prognostic and predictive significance in breast cancer. $\mathrm{Br} J$ Cancer 100: 959-970, 2009.

13. Sen S, Zhou H, Zhang RD, et al: Amplification/overexpression of a mitotic kinase gene in human bladder cancer. J Natl Cancer Inst 94: 1320-1329, 2002.

14. Tanaka E, Hashimoto Y, Ito T, et al: The clinical significance of Aurora-A/STK15/BTAK expression in human esophageal squamous cell carcinoma. Clin Cancer Res 11: 1827-1834, 2005.

15. Reiter R, Gais P, Jutting U, et al: Aurora kinase A messenger RNA overexpression is correlated with tumor progression and shortened survival in head and neck squamous cell carcinoma. Clin Cancer Res 12: 5136-5141, 2006.

16. Guan Z, Wang XR, Zhu XF, et al: Aurora-A, a negative prognostic marker, increases migration and decreases radiosensitivity in cancer cells. Cancer Res 67: 10436-10444, 2007.

17. Ogawa E, Takenaka K, Katakura H, et al: Perimembrane Aurora-A expression is a significant prognostic factor in correlation with proliferative activity in non-small cell lung cancer (NSCLC). Ann Surg Oncol 15: 547-554, 2008.

18. Sundar SS and Ganesan TS: Role of lymphangiogenesis in cancer. J Clin Oncol 25: 4298-4307, 2007.

19. Ferrara N: Pathways mediating VEGF-independent tumor angiogenesis. Cytokine Growth Factor Rev 21: 21-26, 2010.

20. Kopfstein L, Veikkola T, Djonov VG, et al: Distinct roles of vascular endothelial growth factor-D in lymphangiogenesis and metastasis. Am J Pathol 170: 1348-1361, 2007.
21. Halin C, Tobler NE, Vigl B, Brown LF and Detmar M: VEGF-A produced by chronically inflamed tissue induces lymphangiogenesis in draining lymph nodes. Blood 110: 3158-3167, 2007.

22. Ny A, Koch M, Vandevelde W, et al: Role of VEGF-D and VEGFR-3 in developmental lymphangiogenesis, a chemicogenetic study in Xenopus tadpoles. Blood 112: 1740-1749, 2008.

23. Issaka RB, Oommen S, Gupta SK, et al: Vascular endothelial growth factors $\mathrm{C}$ and $\mathrm{D}$ induces proliferation of lymphangioleiomyomatosis cells through autocrine crosstalk with endothelium. Am J Pathol 175: 1410-1420, 2009.

24. Deng J, Liang H, Sun D, Pan Y, Wang B and Guo Y: Vascular endothelial growth factor-D is correlated with hepatic metastasis from gastric cancer after radical gastrectomy. Surgery 146: 896-905, 2009.

25. Vidal O, Metges JP, Elizalde I, et al: High preoperative serum vascular endothelial growth factor levels predict poor clinical outcome after curative resection of gastric cancer. Br J Surg 96: 1443-1451, 2009

26. Juttner S, Wissmann C, Jons T, et al: Vascular endothelial growth factor-D and its receptor VEGFR-3: two novel independent prognostic markers in gastric adenocarcinoma. J Clin Oncol 24: 228-240, 2006.

27. Greene FL, Page DL, Fleming ID and Fritz AG: AJCC Cancer Staging Mannual. Springer-Verlag, New York, p99-103, 2002.

28. Li Y, Qian H, Li X, et al: Adenoviral-mediated gene transfer of Gadd45a results in suppression by inducing apoptosis and cell cycle arrest in pancreatic cancer cell. J Gene Med 11: 3-13, 2009.

29. Livak KJ and Schmittgen TD: Analysis of relative gene expression data using real-time quantitative PCR and the 2[-Delta Delta C(T)] method. Methods 25: 402-408, 2001.

30. Sakakura C, Hagiwara A, Yasuoka R, et al: Tumour-amplified kinase BTAK is amplified and overexpressed in gastric cancers with possible involvement in aneuploid formation. Br J Cancer 84: 824-831, 2001

31. Kamada K, Yamada Y, Hirao T, et al: Amplification/overexpression of Aurora-A in human gastric carcinoma: potential role in differentiated type gastric carcinogenesis. Oncol Rep 12: 593-599, 2004

32. Lassmann S, Danciu M, Muller M, et al: Aurora A is differentially expressed and regulated in chromosomal and microsatellite instable sporadic colorectal cancers. Mod Pathol 22: 1385-1397, 2009.

33. Liu Q, Kaneko S, Yang L, et al: Aurora-A abrogation of p53 DNA binding and transactivation activity by phosphorylation of serine 215. J Biol Chem 279: 52175-52182, 2004.

34. Dar AA, Zaika A, Piazuelo MB, et al: Frequent overexpression of Aurora Kinase A in upper gastrointestinal adenocarcinomas correlates with potent antiapoptotic functions. Cancer 112: $1688-1698,2008$

35. Shen YC, Hu FC, Jeng YM, et al: Nuclear overexpression of mitotic regulatory proteins in biliary tract cancer: correlation with clinicopathologic features and patient survival. Cancer Epidemiol Biomarkers Prev 18: 417-423, 2009.

36. Hung LY, Tseng JT, Lee YC, et al: Nuclear epidermal growth factor receptor (EGFR) interacts with signal transducer and activator of transcription 5 (STAT5) in activating Aurora-A gene expression. Nucleic Acids Res 36: 4337-4351, 2008.

37. Lai CH, Tseng JT, Lee YC, et al: Translational up-regulation of Aurora-A in EGFR-overexpressed cancer. J Cell Mol Med 14: $1520-1531,2010$

38. Landen CN Jr, Lin YG, Immaneni A, et al: Overexpression of the centrosomal protein Aurora-A kinase is associated with poor prognosis in epithelial ovarian cancer patients. Clin Cancer Res 13: 4098-4104, 2007.

39. Mendiola M, Barriuso J, Marino-Enriquez A, et al: Aurora kinases as prognostic biomarkers in ovarian carcinoma. Hum Pathol 40: 631-638, 2009.

40. Zhang W, Wang J, Liu SJ, Hua W and Xin XY: Correlation between Aurora-A expression and the prognosis of cervical carcinoma patients. Acta Obstet Gynecol Scand 88: 521-527, 2009.

41. Nadler Y, Camp RL, Schwartz C, Rimm DL, Kluger HM and Kluger Y: Expression of Aurora A (but not Aurora B) is predictive of survival in breast cancer. Clin Cancer Res 14: 4455-4462, 2008.

42. Shang X, Burlingame SM, Okcu MF, et al: Aurora A is a negative prognostic factor and a new therapeutic target in human neuroblastoma. Mol Cancer Ther 8: 2461-2469, 2009. 
43. Scarpini S, Roupret M, Renard-Penna R, Camparo P, Cussenot O and Comperat E: Impact of the expression of Aurora-A, p53, and Mib-1 on the prognosis of urothelial carcinomas of the upper urinary tract. Urol Oncol: Feb 27, 2010 (Epub ahead of print).

44. Shida A, Fujioka S, Ishibashi Y, et al: Prognostic significance of vascular endothelial growth factor D in gastric carcinoma. World J Surg 29: 1600-1607, 2005.

45. Hata T, Furukawa T, Sunamura M, et al: RNA interference targeting aurora kinase a suppresses tumor growth and enhances the taxane chemosensitivity in human pancreatic cancer cells. Cancer Res 65: 2899-2905, 2005.
46. Lassmann S, Shen Y, Jutting U, et al: Predictive value of Aurora-A/STK15 expression for late stage epithelial ovarian cancer patients treated by adjuvant chemotherapy. Clin Cancer Res 13: 4083-4091, 2007.

47. Steeghs N, Eskens FA, Gelderblom H, et al: Phase I pharmacokinetic and pharmacodynamic study of the aurora kinase inhibitor danusertib in patients with advanced or metastatic solid tumors. J Clin Oncol 27: 5094-5101, 2009.

48. Shimomura T, Hasako S, Nakatsuru Y, et al: MK-5108, a highly selective Aurora-A kinase inhibitor, shows antitumor activity alone and in combination with docetaxel. Mol Cancer Ther 9: 157-166, 2010. 